\title{
In-situ analysis of the structural formation process of liquid-crystalline epoxy thermosets by simultaneous SAXS/WAXS measurements using synchrotron radiation
}

\author{
Miyuki Harada ${ }^{1}$, Junichiro Ando ${ }^{1}$, Seiya Hattori ${ }^{1}$, Shinichi Sakurai ${ }^{2}$, Naoki Sakamoto ${ }^{3}$, Terumasa Yamasaki ${ }^{3}$, \\ Hiroyasu Masunaga ${ }^{4}$ and Mitsukazu Ochi ${ }^{1}$
}

\begin{abstract}
We present a mechanistic study of the curing process of liquid-crystalline epoxy resins by the simultaneous measurement of small-angle X-ray scattering/wide-angle X-ray scattering using synchrotron radiation. The systems studied consist of a diglycidyl ether of terephthalydene-bis(4-amino-3-methylphenol) epoxy component and three types of aromatic amine-type curing agents 4,4'-diaminodiphenylmethane, 4,4'-diaminodiphenylethane and $\mathrm{m}$-phenylenediamine. The systems studied all exhibit similar thermosetting behaviors; first, the epoxide group reacts with an amine moiety of the curing agent, followed by the formation of the nematic domains in the early stage of the process. The nematic-to-smectic conversion proceeds after an incubation period, implying that a critical amount of nematic volume is necessary for phase transition. We found that the transformation continues beyond the conversion of $80 \%$ of the epoxide-amine bond formation and the corresponding entire polymer network formation. The volume fraction of smectic phases after completion of the reaction differs according to the structure of the curing agents. The study clarifies the mechanistic sequence and the preference for smectic phase formation both in terms of time sequence and the dependence of the structure on the curing agents.
\end{abstract}

Polymer Journal (2013) 45, 43-49; doi:10.1038/pj.2012.196; published online 21 November 2012

Keywords: epoxy; in situ; liquid crystalline; SAXS; time-resolved

\section{INTRODUCTION}

Epoxy resins are widely used as adhesives, coatings, paints and electrical insulation materials in industrial fields. Recently, the strong demand for highly functional and high-performance epoxy resins for use in electrical insulation materials has increased. Liquid-crystalline (LC) epoxy resin is well-known as one of the particular epoxy resins that shows an LC phase temperature region. LC epoxy resins have been studied for their unique and excellent properties such as high $\mathrm{Tg}{ }^{1-2}$ fracture toughness ${ }^{3-11}$ and thermal conductivity. ${ }^{12-13}$ The syntheses of many novel LC epoxy resins have been reported and the liquid crystallinity of the epoxy monomers and cured resins have been investigated as well. ${ }^{14-21}$ However, so far, few reports have been published on the ordered structure and physical properties of cured resins.

In contrast, we have already reported how highly ordered LC epoxy network polymers influence their thermal and mechanical properties. One of our important objectives is to design novel highly functional and high-performance network polymers by the introduction of high regularity to the network chains. In our previous report, ${ }^{8-9}$ we discussed the relationship between the domain diameter and fracture toughness in the polydomain cured resins with smectic or nematic LC phase structures. We then concluded that fracture toughness increases with the domain diameter. However, the mechanism of the LC structure formation during the curing process has not been clarified in detail, because of the complexity of the simultaneous progress of the LC structure formation and 3D network structure formation.

In this study, we studied the LC structure formation process in epoxy resins with simultaneous measurement of small- and wideangle X-ray scattering (SAXS/WAXS) using synchrotron radiation. ${ }^{22}$ Three types of LC epoxy curing systems were studied with varying aromatic amine-type curing agents with different chemical structures (4,4'-diaminodiphenylmethane (DDM), 4,4'-diaminodiphenylethane (DDE), and m-phenylenediamine (m-PDA)). The bonding geometry of the curing agent strongly affects the orientation of mesogenic groups in the network chains. In addition, we discuss the relationship between the LC structure formation process based on SAXS/WAXS results and the reaction of epoxy groups and gel formation. 


\section{EXPERIMENTAL PROCEDURE}

\section{Samples}

The chemical structure of the epoxy material and curing agents used in this study are shown in Figure 1. The details of the synthesis of the diglycidyl ether of terephthalydene-bis(4-amino-3-methylphenol) (DGETAM) are shown elsewhere. ${ }^{6}$ All of the agents listed in Figure 1 are powder at room temperature. Equimolar amounts of the epoxy resin and one of the curing agents were mixed and pounded in a mortar.

\section{SAXS and WAXS measurements}

In-situ measurements of the formation of the liquid-crystalline structure during the curing process have been carried out with simultaneous SAXS and WAXS measurements at the BL03XU ${ }^{22}$ beam line in SPring- 8 . The typical measurement procedure involves the following steps: (1) the sample powder mixture is placed in the cell; (2) curing is commenced by the temperaturejump method that involves inserting the sample cell into the heater block placed in the incident X-ray beam; and (3) the time-resolved measurements for SAXS, WAXS and the transmission, $T$, of the sample for the incident X-ray are started when the sample cell is transferred into the heater block. The schematic illustration of the temperature-jump experiment is shown in Figure 2. The wavelength $\lambda$ of the incident X-ray beam was $0.1 \mathrm{~nm}$, and the sample-todetector distances were $60 \mathrm{~mm}$ for WAXS and $600 \mathrm{~mm}$ for SAXS. The accumulation time of each shot and the time interval between the measurements were 500 and $4500 \mathrm{~ms}$, respectively. The two-dimensional (2D) X-ray scattering patterns were detected by the flat-panel detector (Hamamatsu Photonics, Shizuoka, Japan, C9827DK-10) for WAXS and the image intensifier with the cooled CCD (II CCD; Hamamatsu Photonics, V7739P + ORCA R2) for SAXS. The SAXS and WAXS patterns obtained were averaged circularly and corrected for absorption, the sample thickness, $t$, the scattering from the empty cell, and the dark current of the detectors. As the sample investigated here is powder in the initial state and then melts and forms resin after the temperature-jump, the sample thickness inevitably changes with time. The $t$ at each data-collection point was estimated by the Lambert-Beer law in equation 1:

$$
T=\exp (-\mu t)
$$

where $\mu$ is the linear absorption coefficient for the X-ray with $\lambda=0.1 \mathrm{~nm}$ and is assumed to be independent of the temperature and chemical conversion. Two types of the sample cells were used here, referred as Type-I cell and Type-II cell. The time changes of the sample temperature in the cells after the temperaturejump to $170{ }^{\circ} \mathrm{C}$, measured by inserting thermocouples directly into the sample, are shown in Figure 3. As the cell size of the Type II cell is smaller than that of the Type I cell, the sample temperature of the Type II cell reaches $170{ }^{\circ} \mathrm{C} \sim 90 \mathrm{~s}$ earlier than the Type I cell.

\section{Measurement of chemical conversion}

The chemical conversion of the cures was confirmed using FT-IR (Fourier transform-infrared) spectroscopy (SPECTRUM 2000, Perkin-Elmer Inc., Waltham, MA, USA) and the KBr method. The resolution of the IR spectrum was $4 \mathrm{~cm}^{-1}$ and the spectra were collected after 4 scans. The peak at $1600 \mathrm{~cm}^{-1}$, which is derived from the skeletal vibration of the aromatic ring was used as the internal standard.

\section{Measurement of the gel fraction}

The gel fraction was measured using THF as the solvent. The solvent was changed every $0.5 \mathrm{~h}$ at room temperature for $5 \mathrm{~h}$ and every $1 \mathrm{~h}$ at $60^{\circ} \mathrm{C}$ for $10 \mathrm{~h}$. The samples were dried under vacuum at $80^{\circ} \mathrm{C}$ for $3 \mathrm{~h}$. The gel fraction of the samples was obtained by dividing the gel weight by their original weight.

\section{Epoxy resin}

Terephthalylidene-bis-(4-amino-3-methylphenol)DGE (DGETAM, Mw = 456, C 169 N 212 I)<smiles>Cc1cc(OCC2CO2)ccc1N=Cc1ccc(C=Nc2ccc(OCC3CO3)cc2C)cc1</smiles>

\section{Curing Agents}
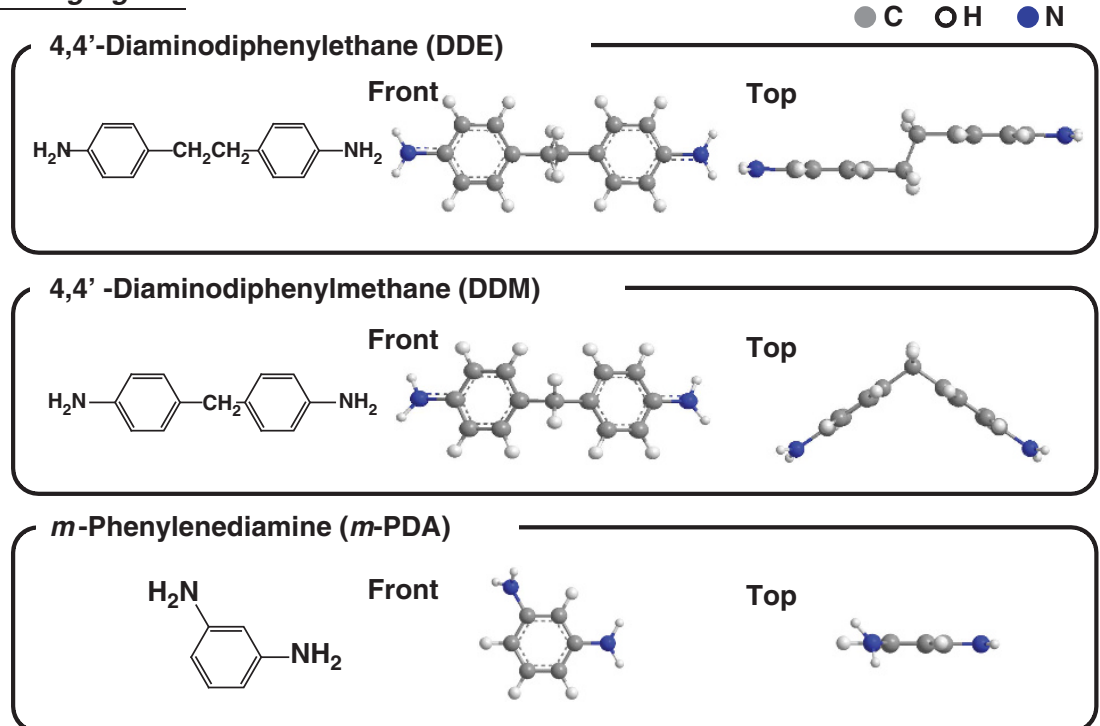

Top

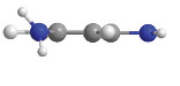

Figure 1 Chemical structures of epoxy resin and curing agents. 

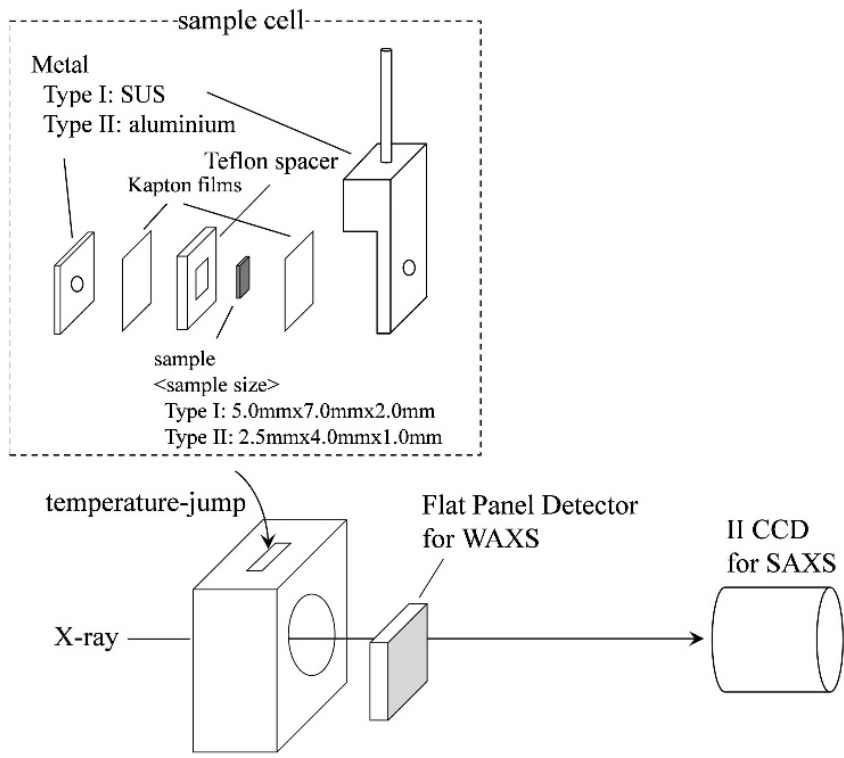

heater block

Figure 2 Schematic illustration of the in-situ small-angle X-ray scattering (SAXS) and wide-angle X-ray scattering (WAXS) simultaneous measurement set up for the temperature-jump experiment.

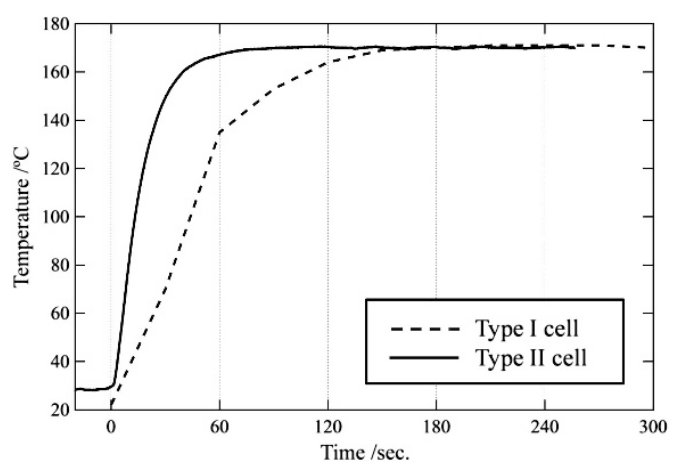

Figure 3 Time changes of the sample temperature in the cells, measured by inserting thermocouples directly into the sample directly, after the temperature-jump to $170^{\circ} \mathrm{C}$.

\section{RESULTS AND DISCUSSION}

\section{Formation mechanism of the liquid-crystalline structure in} the curing process

Figure 4 shows the time-dependent WAXS and SAXS profiles of the DGETAM/DDE system after the temperature-jump to $170^{\circ} \mathrm{C}$. The abscissa indicates the magnitude of the scattering vector $(q)$, which is defined in equation 2 ,

$$
q=4 \pi \sin (\theta / 2) / \lambda
$$

where $\theta$ denotes the scattering angle. The experiment was performed by using a Type I cell. In the WAXS profiles, sharp reflection peaks from the crystalline phase appear only in the very early stage of the measurements right after the temperature-jump, and its intensity deteriorated rapidly owing to the melting of crystalline moieties. Finally, all peaks disappeared. In contrast, a broad peak appears at $q=14 \mathrm{~nm}^{-1}$ (corresponding to a Bragg Spacing $(d)$ of $0.44 \mathrm{~nm}$ ) and shows no obvious change after $3 \mathrm{~min}$, though the peak position slightly shifts toward smaller $q$. Using a polarized optical microscope, we confirmed that the system forms a nematic phase after the melting of the crystalline components in the starting materials. Moreover, it was found that the sample transitions to the smectic phase after 6 min as described below. Figure 4a implies that the transition from the nematic-to-smectic phase does not change the WAXS profile. In this study, the 2D SAXS and WAXS patterns (not shown here) are isotropic, meaning the liquid-crystalline phases have random orientations in the illuminated area of the incident X-ray. In our previous study, we reported that the orientation can be aligned by a $10 \mathrm{~T}$ magnetic field. ${ }^{10}$ The study revealed that the $2 \mathrm{D}$ X-ray scattering pattern of the cured sample showed the spots due to the smectic layers in the direction of the magnetic field, observed in a SAXS region, and the arches in the perpendicular direction, in a WAXS region, corresponding to the peak in Figure 4a. This indicates that the WAXS peak of this system, that is, the broad peak in Figure $4 \mathrm{a}$, is assigned to the correlation between the neighboring mesogenic groups in the liquid-crystalline phase, not because of the molecular length of the mesogen. The time evolution of the WAXS profile is well understood; the nematic phase formation has almost simultaneously completed upon melting of the crystalline components. In contrast, the SAXS profiles in Figure 4b show no peak before $6 \mathrm{~min}$. The peak due to the smectic layers composed of the mesogenic groups appears at $q=2.3 \mathrm{~nm}^{-1}(d=2.7 \mathrm{~nm})$ at $6 \mathrm{~min}$, and the intensity increases along with the time duration. Here, we note that the peak shape of the SAXS profiles is not very sharp, and the higher-order corresponding peaks are not observed. This implies that the domain of the liquid-crystalline phase is relatively small or distorted.

Figure 5 shows the IR spectra at various curing times. The intensity of the peak at $910 \mathrm{~cm}^{-1}$, which derived from the epoxy group, gradually decreased, and that at $3200-3400 \mathrm{~cm}^{-1}$, which is derived from the hydroxyl group, increased with the progress of the ring opening reaction of the epoxy.

Figure 6 shows the time dependence of the peak intensity of the SAXS profiles obtained from Figure $4 \mathrm{~b}$, the conversion of the epoxy group calculated from the IR spectra and the gel fraction. With this evolution of the SAXS profile, we reason that the smectic structure appears at $\sim 6 \mathrm{~min}$ and the formation saturates at $\sim 15 \mathrm{~min}$. We notice that the conversion of the epoxy group increases from the time just after the temperature-jump, and it reaches $96 \%$ at ca $30 \mathrm{~min}$. Similarly, the gel formation starts at $5 \mathrm{~min}$ when the chemical conversion is $\sim 60 \%$, and the fraction increases rapidly.

It should be mentioned that the curing experiments for WAXS/ SAXS measurements and those for FT-IR and gel fraction were performed separately with different heating devices. The small difference in the temperature or the heating rate of the samples may lead to the difference in the time changes of the reaction and the formation of the liquid-crystalline structure. To confirm this point, the gel fraction of the sample, cured for $10 \mathrm{~min}$ in the chamber used for the WAXS and SAXS, was measured. As a result, the value is equivalent to that at $10 \mathrm{~min}$ in Figure 6, indicating that we can compare the time evolution of the data in Figure 6 with each other.

Figures 4, 5 and 6 show that the formation of the liquid-crystalline structure after the temperature-jump proceeds as follows: (1) the crystalline phase of DGETAM/DDE melts, and the nematic structure forms (0-3 min); (2) the nematic phase lasts for $6 \mathrm{~min}$; (3) the transition from the nematic to the smectic phase starts at $6 \mathrm{~min}$, indicating that there is an induction period; and (4) the formation of the smectic structure proceeds until $15 \mathrm{~min}$ has passed. In contrast, the chemical conversion reaches $65 \%$ when the formation of the smectic phase starts, indicating that the transition from the nematic to the smectic phase starts under the existing network structure, not in the state in which the conversion is low, and hence the mesogenic group still possesses a certain amount of flexibility. Moreover, the 

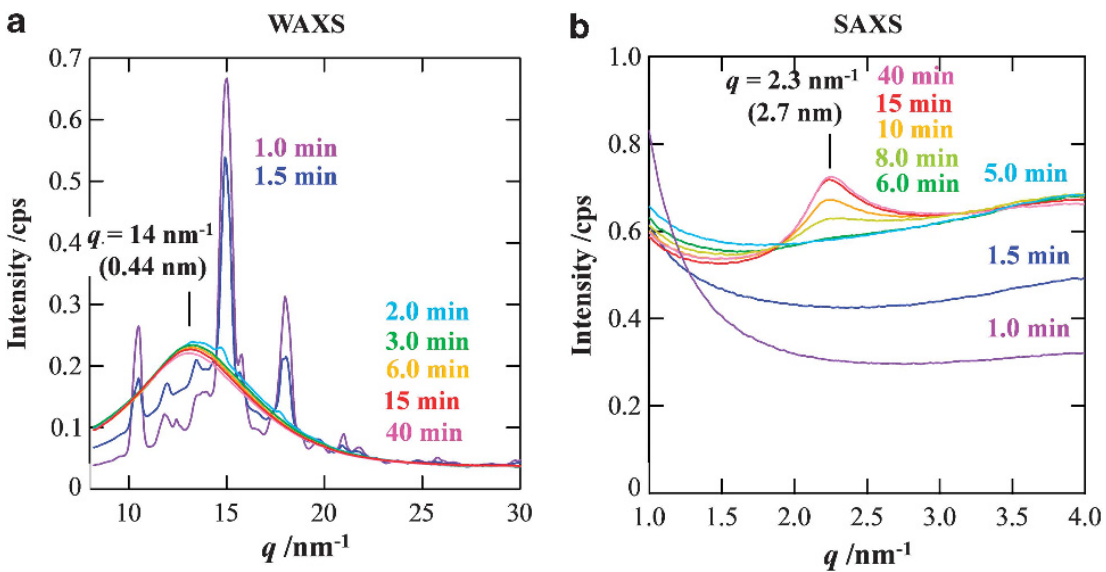

Figure 4 (a) Wide-angle X-ray scattering (WAXS) and (b) small-angle X-ray scattering (SAXS) profiles of the diglycidyl ether of terephthalydene-bis(4-amino3-methylphenol) (DGETAM)/4,4'-diaminodiphenylethane (DDE) after the temperature-jump to $170{ }^{\circ} \mathrm{C}$.



Figure 5 Fourier transform-infrared (FT-IR) spectra for the curing process of the diglycidyl ether of terephthalydene-bis(4-amino-3-methylphenol) (DGETAM)/4,4'-diaminodiphenylethane (DDE) at $170^{\circ} \mathrm{C}$.

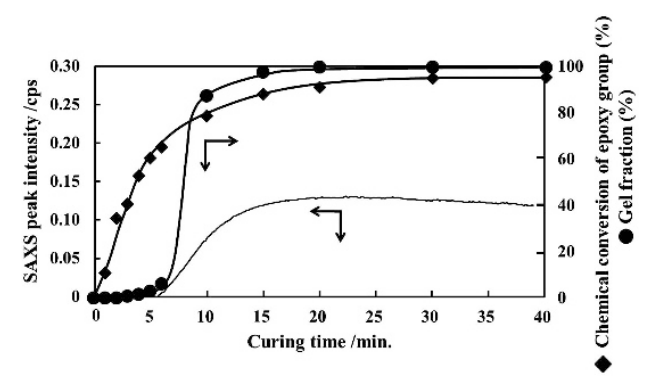

Figure 6 Time evolution of the peak intensity in small-angle X-ray scattering (SAXS) profiles, chemical conversion of the epoxy group and the gel fraction after the temperature-jump of the diglycidyl ether of terephthalydene-bis(4amino-3-methylphenol) (DGETAM)/4,4'-diaminodiphenylethane (DDE) to $170^{\circ} \mathrm{C}$.

formation of the smectic structure proceeds even at $10 \mathrm{~min}$, when the cross-linking structure is supposed to have grown sufficiently because the chemical conversion and the gel fraction are 80 and $85 \%$, respectively. Under the existing network structure, the resulting local translational motion of the mesogenic group between the crosslinking points induces the domain structure to transform from the nematic to the smectic phase. It is conceivable that such a structural transformation under the restricted freedom of motion results in the long time duration of $\sim 10 \mathrm{~min}$ for the completion of the smectic phase structure.

In the system studied here, it is presumed that all the nematic phase does not completely transform into the smectic phase. In a general liquid-crystalline system in which a cross-linking reaction does not occur, all of the nematic structures should transition to the smectic structures if the smectic phase is more stable than the nematic from the point of view of thermodynamics. However, in this system, some mesogenic groups in the nematic phase cannot align with each other owing to topological restrictions, that is, pinning with cross-linking points, and they may retain the nematic structure even after a sufficient curing time at $170{ }^{\circ} \mathrm{C}$, though the smectic phase is expected to be more stable than the nematic one if they are not pinned. The coexistence of the smectic and the nematic phases after curing will also be discussed in the next section. Here, it should be mentioned that the formation of the smectic phase starts after gelation, indicating that the reaction of DGETAM and curing agents, that is, the pinning of mesogenic groups collectively, promotes the transition. In contrast, the crosslinking prevents the motion of the mesogen and suppresses the formation of the smectic structure. Such a dual effect of crosslinking is unique to the current system, and the degree of transition from the nematic to the smectic phase is determined by this competition.

In Figure 6, the SAXS peak intensity decreases gradually after $15 \mathrm{~min}$. This means that the order of the smectic structure decreases gradually with progress of the chemical conversion from 97 to $100 \%$. The polymer network tightening may lead to distortion of the smectic phase; hence, a part of the smectic phase becomes disordered.

\section{Curing temperature dependence of structural formation}

Figure 7 shows the time evolution in the peak intensity of the SAXS profiles after the temperature-jump to 170 and $200{ }^{\circ} \mathrm{C}$ for the DGETAM/DDE. The data at $170^{\circ} \mathrm{C}$ are the same as those in Figure 6. Figure 7 indicates that the SAXS peak intensity at $200^{\circ} \mathrm{C}$ increases rapidly at $3 \mathrm{~min}$, and saturates at $8 \mathrm{~min}$. The trend is essentially the same as that at $170{ }^{\circ} \mathrm{C}$, though the time-scale at $200{ }^{\circ} \mathrm{C}$ is shorter than that at $170^{\circ} \mathrm{C}$, meaning that the formation of the smectic phase at $200^{\circ} \mathrm{C}$ proceeds with the same mechanism conceived above.

In Figure 7, it should be noted that the peak intensities at the saturated points are different, such that the peak intensity at $200^{\circ} \mathrm{C}$ is smaller than that at $170^{\circ} \mathrm{C}$. As the scattering contrast of the smectic structure at $170{ }^{\circ} \mathrm{C}$ is considered to be not very different from that at 


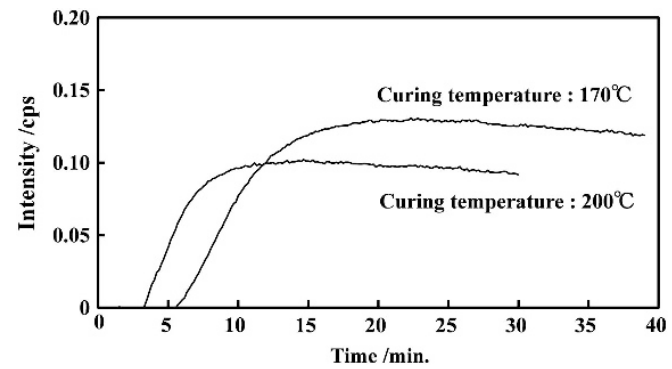

Figure 7 Time evolution of the small-angle X-ray scattering (SAXS) peak intensity of the diglycidyl ether of terephthalydene-bis(4-amino-3methylphenol) (DGETAM)/4,4'-diaminodiphenylethane (DDE) after the temperature-jump to 200 and $170^{\circ} \mathrm{C}$.

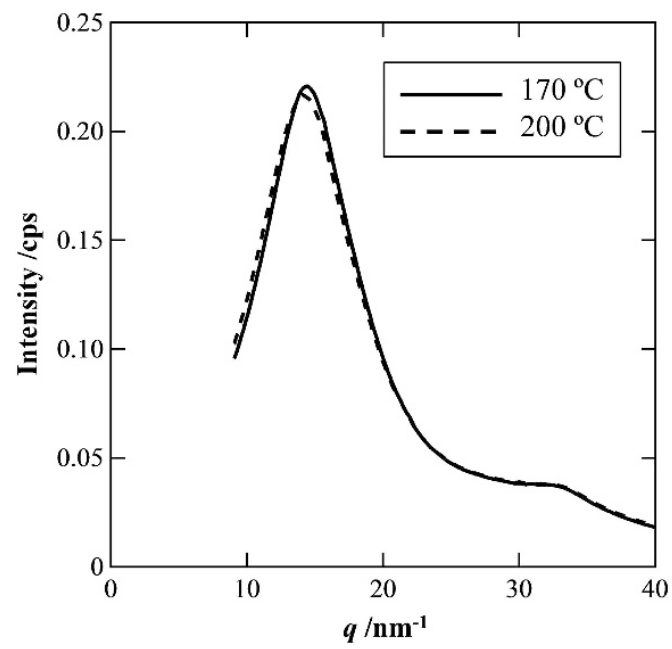

Figure 8 Wide-angle $\mathrm{X}$-ray scattering (WAXS) profiles at $30 \mathrm{~min}$ after the temperature-jump to 170 and $200^{\circ} \mathrm{C}$ for diglycidyl ether of terephthalydenebis(4-amino-3-methylphenol) (DGETAM)/4,4'-diaminodiphenylethane (DDE).

$200{ }^{\circ} \mathrm{C}$, the volume fraction of the smectic phase at $200^{\circ} \mathrm{C}$ is smaller than that at $170{ }^{\circ} \mathrm{C}$. In contrast, the WAXS profiles at $30 \mathrm{~min}$ after the temperature-jump to $170{ }^{\circ} \mathrm{C}$ and $200^{\circ} \mathrm{C}$ are almost equivalent, as shown in Figure 8. The figure shows that the volume fractions of the liquid-crystalline phase cured at 170 and $200{ }^{\circ} \mathrm{C}$ are the same, though we cannot determine whether the phase is smectic or nematic, because the WAXS profiles for both phases show no differences as mentioned in Figure 4a. These results imply that the smectic and the nematic phases coexist and that the fraction of these phases differs according to the curing temperature. The fast reaction at $200{ }^{\circ} \mathrm{C}$ may result in an inhomogeneous distribution of the cross-linking points. Consequently, the formation of the smectic phase is hindered in the area where the density of the cross-linking points is high and then the motion of the mesogens is restricted.

Effect of the chemical structure of the curing agents on the formation of the smectic phase

Figure 9 shows the time evolution in the SAXS peak intensity after the temperature-jump to $170{ }^{\circ} \mathrm{C}$ for the systems with different curing agents. These were measured with the small sample cell, Type II, in which the sample reaches $170^{\circ} \mathrm{C}$ within $80 \mathrm{~s}$ after the temperaturejump, that is, $\sim 1.5 \mathrm{~min}$ faster than in the Type I cell (see Figure 3 ). Consequently, the formation of the smectic phase of the DGETAM/
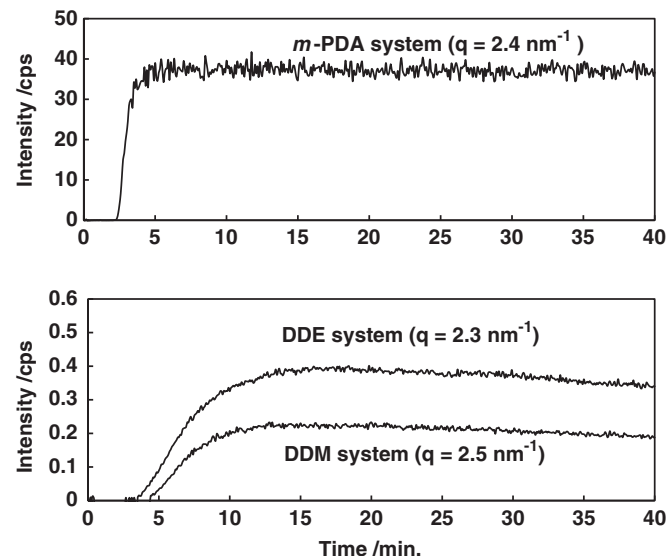

Figure 9 Time evolution of the small-angle X-ray scattering (SAXS) peak intensity after the temperature-jump to $170^{\circ} \mathrm{C}$ for the mixtures of the diglycidyl ether of terephthalydene-bis(4-amino-3-methylphenol) (DGETAM) and the curing agent shown in Figure 1.

DDE in Figure 9 begins 1.5 min earlier than the results shown in Figure 6.

All of the curves show induction periods, indicating that the reaction and the phase transitions sequences towards the smectic phase are quite similar in these systems. It should be noted, though, that the saturated intensities are very different according to the curing agents in Figure 9. The SAXS profiles of the samples with m-PDA, $\mathrm{DDE}$ and DDM at $40 \mathrm{~min}$ after the temperature-jump to $170{ }^{\circ} \mathrm{C}$ are shown in Figure 10. The intensities and the sharpness of the peaks are on the order of $\mathrm{m}-\mathrm{PDA}>>\mathrm{DDE}>\mathrm{DDM}$. The smectic structure formed by the liquid-crystalline epoxy resins, studied here is composed of mesogenic layers in which benzene rings stack with each other and the gap between the layers is filled primarily by the nonmesogenic components, as we show schematically in Figure 11. When DGETAM and the curing agent form the smectic structures, the curing agent and DGETAM should take part in the formation of mesogenic layers. Therefore, the structures of the curing agents affect the stability of the smectic structures being formed from an enthalpic point of view.

In Figure 1, we presented molecular models for DDE, DDM and $\mathrm{m}$-PDA. It should be noted that m-PDA, DDM and DDE have planar, bending and pseudo-planar structures at the methylene carbons, respectively. As the intensity of smectic peaks in the SAXS profiles correspond to the alignment of the mesogenic layer units, the planar structure of the curing agent seems preferable for the formation of smectic domains. Conceivably, in such a structure, mesogenic units form densely stacked, well-ordered layers with a high volume fraction of the smectic phase, resulting in the SAXS peak of the m-PDA system being much larger and sharper than those of DDE and DDM systems. The DDE structure is closer to planar than the DDM structure and then the peak intensity of the DDE system is in between the peak intensities of the m-PDA and DDM systems.

In Figure 9, we note that the peak intensity of the m-PDA does not decrease, though those of the DDE and DDM systems gradually decrease with time after they saturate, as described in Figure 6. This may be related to the stability of the smectic phase, as mentioned above. The layered structure consisting of the well-stacked benzene rings of DGETAM and m-PDA may be able to prevent being disordered as the distortion increases with the progress of the chemical conversion. 


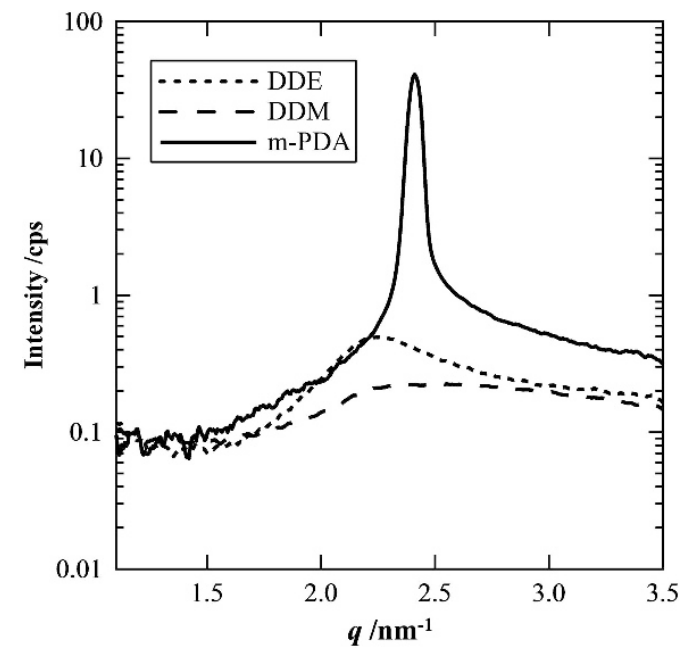

Figure 10 Small-angle $\mathrm{X}$-ray scattering (SAXS) profiles of the m-phenylenediamine (m-PDA), 4,4'-diaminodiphenylethane (DDE) and 4,4'-diaminodiphenylmethane (DDM) systems at $40 \mathrm{~min}$ after the temperature-jump to $170^{\circ} \mathrm{C}$.

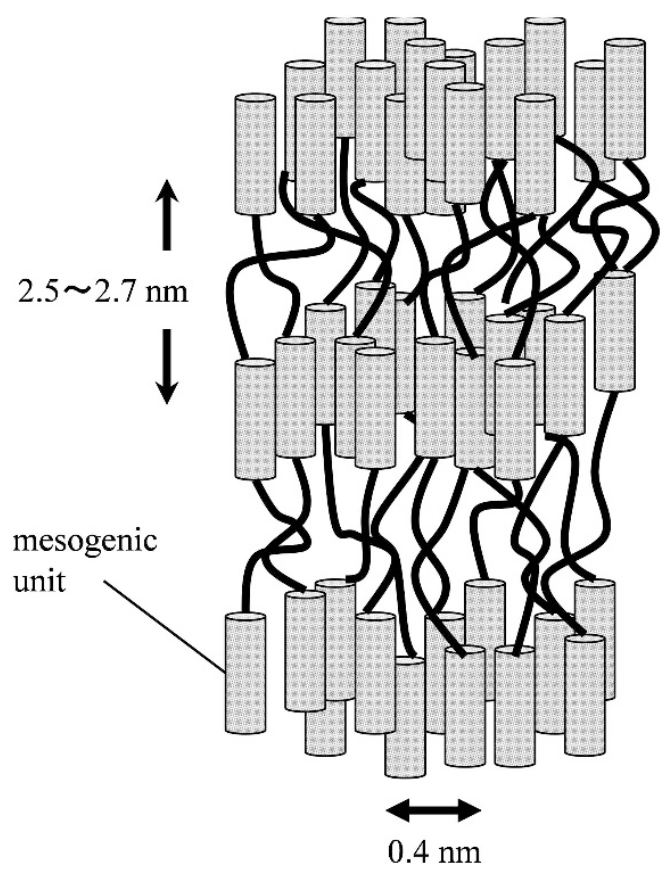

Figure $11 \mathrm{~A}$ schematic representation of the neighboring mesogenic layers. Cylinders show the mesogenic units.

\section{CONCLUSIONS}

In-situ SAXS/WAXS simultaneous measurements have successfully clarified the structural formation mechanism of liquid-crystalline epoxy resins in the curing process: (1) the crystalline phase of the starting materials melts, and the nematic structure forms just after the temperature-jump; (2) the nematic phase remains during an induction period; (3) the transition from the nematic to the smectic phase starts and proceeds until the smectic phase entirely coverers the regions where it is possible to form the smectic structure topologically; and (4) the order of the smectic structure decreases gradually as the chemical conversion increases, and accordingly, the polymer network becomes tighter. Noticeably, the time evolution of the chemical conversion obtained by FT-IR and the gel fraction revealed that the transition from the nematic to the smectic phase starts under the existing network structure, not in the state in which the conversion is low, and hence the mesogenic group still possesses a good mobility. Moreover, the transition from the nematic to the smectic phase continues even at the time when the chemical conversion and the gel fraction are more than $80 \%$ and $85 \%$, respectively. Under the existing network structure, the local translational motion of the mesogenic group between the cross-linking points enables the transformation from the nematic to the smectic phase.

The temperature-jump measurements to different temperatures indicate that the volume fraction of the smectic phase formed at the higher temperature is smaller than that formed at the lower temperature. The inhomogeneous distribution of the cross-linking point, made at the high curing temperature, may hinder the formation of the smectic phase.

The comparison of the SAXS profile of the m-PDA, DDE and DDM systems after curing revealed that the steric structure of the curing agents strongly affected the order and the volume fraction of the smectic phase formed by the curing process, that is, those in the m-PDA system are much higher than the DDE and DDM systems. As both m-PDA and DGETAM have a planar structure, they may easily form densely stacked mesogenic layers, leading to the well-ordered high volume fraction of the smectic phase.

\section{CONFLICT OF INTEREST}

The authors declare no conflict of interest.

\section{ACKNOWLEDGEMENTS}

The simultaneous SAXS and WAXS measurements were carried out at the second hutch of SPring-8 BL03XU constructed by the Consortium of Advanced Softmaterial Beam line (FSBL), with the proposal number 2010A7201, 2010B7251, 2011A7201 and 2011B7251.

1 Ochi, M., Tsuyuno, N., Sakaga, K., Nakanishi, Y. \& Murata, Y. Effect of network structure on thermal and mechanical properties of biphenol-type epoxy resins cured with phenols. J. Appl. Polym. Sci. 56, 1161-1167 (1995).

2 Ochi, M., Shimizu, Y., Nakanishi, Y. \& Murata, Y. Effect of the network structure on thermal and mechanical properties of mesogenic epoxy resin cured with aromatic amine. J. Polym. Sci. B Polym. Phys. 35, 397-405 (1997).

3 Sue, H. J., Earls, J. D. \& Hefner, Jr R. E. Fracture behaviour of liquid crystal epoxy resin systems based on the diglycidyl ether of 4,4'-dihydroxy- $\alpha$-methylstilbene and sulphanilamide: Part I Effects of curing variations. J. Mater. Sci. 32, 4031-4037 (1997).

4 Ortiz, C., Belenky, L., Ober, C. K. \& Kramer, J. Microdeformation of a polydomain, smectic liquid crystalline thermoset. J. Mater. Sci. 35, 2079-2086 (2000).

5 Ortiz, C., Kim, R., Rodighiero, E., Ober, C. K. \& Kramer, E. J. Deformation of a polydomain, liquid crystalline epoxy-based thermoset. Macromolecules 31 , 4074-4088 (1998)

6 Harada, M., Aoyama, K. \& Ochi, M. Fracture mechanism of liquid-crystalline epoxy resin systems with different phase structures. J. Polym. Sci. B Polym. Phys. 42, 4044-4052 (2004).

7 Harada, M., Watanabe, Y., Tanaka, Y. \& Ochi, M. Thermal properties and fracture toughness of a liquid-crystalline epoxy resin cured with an aromatic diamine crosslinker having a mesogenic group. J. Polym. Sci. B Polym. Phys. 44, 2486-2494 (2006).

8 Harada, M., Sumitomo, K., Nishimoto, Y. \& Ochi, M. Relationship between fracture toughness and domain size of liquid-crystalline epoxy resins having polydomain structure. J. Polym. Sci. B Polym. Phys. 47, 156-165 (2009).

9 Harada, M., Okamoto, N. \& Ochi, M. Fracture toughness and fracture mechanism of liquid-crystalline epoxy resins with different polydomain structures. J. Polym. Sci. B Polym. Phys. 48, 2337-2345 (2010).

10 Harada, M., Ochi, M., Tobita, M., Kimura, T., Ishigaki, T., Shimoyama, N. \& Aoki, H. Thermomechanical properties of liquid-crystalline epoxy networks arranged by a magnetic field. J. Polym. Sci. B Polym. Phys. 42, 758-765 (2004).

11 Potitie, L., Torro, F., Tessier, M., Davidson, P. \& Fradet, A. Investigation of anisotropic epoxy-amine thermosets synthesised in a magnetic field. Liquid Crystal 35, 913-924 (2008).

12 Akatsuka, M. \& Takezawa, Y. Study of high thermal conductive epoxy resins containing controlled high-order structures. J. Appl. Polym. Sci. 89, 2464-2467 (2003). 
13 Harada, M., Ochi, M., Tobita, M., Kimura, T., Ishigaki, T., Shimoyama, N. \& Aoki, H. Thermal-conductivity properties of liquid-crystalline epoxy resin cured under a magnetic field. J. Polym. Sci. B Polym. Phys. 41, 1739-1743 (2003).

14 Liu, L., Gao, J., Du, Y. \& Chai, Z. Synthesis and characterization of the two novel liquid crystalline epoxy resins based on bisphenol-s mesogen. J. Appl. Polym. Sci. 110, 3671-3677 (2008).

15 Lee, J. \& Jang, J. The effect of mesogenic length on the curing behavior and properties of liquid crystalline epoxy resins. Polymer 47, 3036-3042 (2006).

16 Liu, G., Zhou, B., Zhao, D., Li, Q. \& Gao, J. Novel triaromatic ester mesogenic liquid crystalline epoxy resin containing both methyl substituent and ethoxy flexible spacer: synthesis and curing. Macromol. Chem. Phys. 209, 1160-1169 (2008).

17 Choi, E., Seo, J., Bae, H. \& Lee, J. Synthesis and curing of new aromatic azomethine epoxies with alkoxy side groups. Eur. Polym. J. 40, 259-265 (2004).

18 Lee, J. Y. \& Jang, J. Effect of substituents on the curing of liquid crystalline epoxy resin. J. Polym. Sci. A Polym. Chem. 36, 911-917 (1998).
19 Liu, W. \& Carfagna, C. Synthesis of liquid crystalline epoxy oligomers: effect of molecular weight on the phase behavior. Macromol. Rapid. Commun. 22, 1058-1062 (2001).

20 Castell, P., Galia, M. \& Serra, A. Synthesis of new epoxy liquid-crystalline monomers with azo groups in the central mesogenic core. Crosslinking with amines. Macromol. Chem. Phys. 202, 1649-1657 (2001).

21 Ribera, D., Montecon, A. \& Serra, A. Synthesis and crosslinking of a series of dimeric liquid crystalline epoxy resins containing imine mesogens. Macromol. Chem. Phys. 202, 1658-1671 (2001).

22 Masunaga, H., Ogawa, H., Takano, T., Sasaki, S., Goto, S., Tanaka, T., Seike, T. Takahashi, S., Takeshita, K., Nariyama, N., Ohashi, H., Ohata, T., Furukawa, Y., Matsushita, T., Ishizawa, Y., Yagi, N., Takata, M., Kitamura, H., Sakurai, K., Tashiro, K., Takahara, A., Amamiya, Y., Horie, K., Takenaka, M., Kanaya, T., Jinnai, H., Okuda, H., Akiba, I., Takahashi, I., Yamamoto, K., Hikosaka, M., Sakurai, S., Shinohara, Y., Okada, A. \& Sugihara, Y. Multipurpose soft-material SAXS/WAXS/GISAXS beamline at SPring-8. Polym. J. 43, 471-477 (2011). 